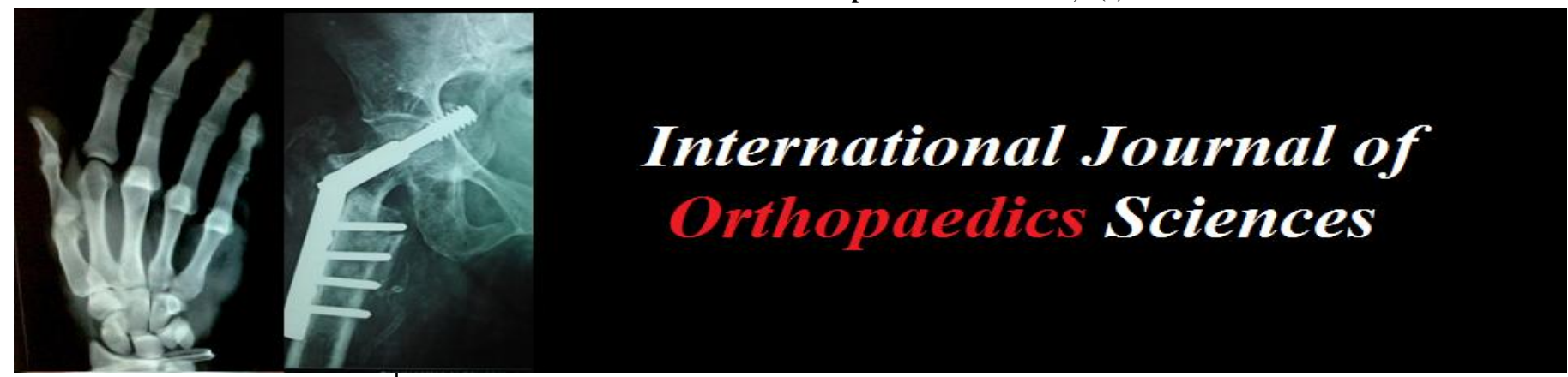

ISSN: $2395-1958$

IJOS 2017; 3(4): 88-94

(C) 2017 IJOS

www.orthopaper.com

Received: 14-08-2017

Accepted: 15-09-2017

Dr. K Harsha Vikram

Kadimi Hospital, Chandanagar, Hyderabad, Telangana, India

Dr. Kadimi Haranadh

Kadimi Hospital, Chandanagar, Hyderabad, Telangana, India

Dr. Guru Prasad Sultanpurkar Kadimi Hospital, Chandanagar, Hyderabad, Telangana, India

Dr. Harshavardhan Raorane Kadimi Hospital, Chandanagar, Hyderabad, Telangana, India
Correspondence

Dr. Guru Prasad Sultanpurkar Kadimi Hospital, Chandanagar, Hyderabad, Telangana, India

\section{A study of long term functional outcome of surgical management of distal femur fractures in adults using locking compression plate in Indian rural set up}

\author{
Dr. K Harsha Vikram, Dr. Kadimi Haranadh, Dr. Guru Prasad \\ Sultanpurkar and Dr. Harshavardhan Raorane
}

DOI: $\underline{\text { https://doi.org/10.22271/ortho.2017.v3.i4b.14 }}$

\section{Abstract}

Objective: To study and analyze the effectiveness of the surgery of the distal femur fractures using Locking Compression Plate based on fracture union, complications, recovery, return to work and overall functional outcome using Neer's criteria.

Introduction: Due to rapid industrialization and growth of the automobile industry, there is increase in the road traffic accidents. Most of the high velocity injuries are associated with fractures occurring at the distal end of the femur. In view of these potential complications and complexity of the injuries at the distal end of femur, many surgeons agree that large numbers of distal femur fractures are to be managed surgically for better functional outcome and lesser complications.

- Internal fixation by using Locking Compression Plates (LCP) is one of the commonly performed surgeries for injuries around the knee because it gives better results in terms of recovery, fracture union, return to work and overall functional outcome

- LCP is a single beam construct where its strength of fixation is equal to sum of all screw-bone interfaces. Its biomechanics works on splinting rather than compression giving a flexible stabilization. furthermore, when applied via a minimally invasive technique, provides prompt healing and less bone resorption due to better preservation of blood supply

- This study will be helpful in understanding and analyzing the benefits and disadvantages of treating the fractures of the distal end of the femur in adults using Locking Compression Plate and functional outcomes of the surgical procedures

Methodology: A group of 40 distal femur fracture patients, treated with open reduction and internal fixation using Locking Compression Plate (LCP) between Feb 2015 to August 2016.

Results: Out of 40 patients excellent results seen in 23 patients $(57.5 \%)$, good results seen in 10 patients (25\%), fair results seen in 3 patients $(7.5 \%)$, and poor results seen in 4 patients $(10 \%)$ using Neer's scoring system.

Keywords: Neer's criteria, distal femur fractures, locking compression plate

\section{Introduction}

The incidence of distal femur fractures is approximately 37 per $1,00,000$ persons ${ }^{[1]}$. Distal femur fractures mainly are the result of two different injury mechanisms. One mechanism is they are often caused by high energy trauma mainly sustained in road traffic accidents. Open injuries with considerable comminution of condyles and metaphysis are frequently seen. Another is the low energy trauma, relating to elderly patients with severe osteoporosis frequently seen as periprosthetic fracture. In elderly patients, extreme osteoporosis represents a particular problem for anchoring the implant ${ }^{[2]}$. Most surgeons agree that distal femur fractures need to be treated operatively to achieve optimal patient outcomes3. Internal fixation with locking plates creates a toggle free, fixed angle construct 4 increases the rigidity of fixation in osteoporotic bone. The LCP is a single beam construct where the strength of its fixation is equal to the sum of all screw-bone interfaces rather than a single screw's axial stiffness or pullout resistance as seen in unlocked plates. Its unique biomechanical function is based on splinting rather than compression resulting in flexible stabilization, avoidance of stress shielding and induction of callus formation. 
Further when it is applied via a minimally invasive technique, it allows for prompt healing, lower rates of infection and reduced bone resorption as blood supply is preserved ${ }^{[5]}$.

The study is undertaken to study and analyze the effectiveness of the surgery of the distal femoral fractures using Locking Compression Plate based on fracture union, complications, recovery, return to work and overall functional outcome using Neer's criteria.

\section{Materials and Methods}

In this study 40 patients with closed distal femur fractures (distal $15 \mathrm{~cm}$ of femur) were studied.

All the cases were treated at KADIMI HOSPITAL between February -2015 \& August-2016 at our institution and followed for a minimum of 1 year. The duration of follow up ranged from 6 months to 18 months.

The following protocol was observed for patients with distal femur fracture on arrival.

1. General, systemic examination as well as local examination of the patient.

2. Assessment to rule out head/ chest/ abdominal/ spinal or pelvic injury.

3. Evaluation of patients in terms of:
a. Age
b. Sex
c. Mode of trauma
d. Period between injury and arrival.

4. Musculo-skeletal examination of patient to rule out associated fractures.

5. Stabilization of patient with intravenous fluids, oxygen and blood transfusion as and when required.

6. Careful assessment of injured limb as regards to neurovascular status.

7. Primary immobilization of involved limb in Thomas splint with a cotton pad below the distal fragment and transport of patient to the department of radio diagnosis in the same.

8. Radiological assessment: Anteroposterior and true lateral views of injured limb including complete knee joint and distal femur/proximal leg.

9. Upper tibial skeletal pin traction with a Steinmann or Denham pin drilled under local anaesthesia followed by continuous traction given over the BB splint was used.

\section{Patient Selection}

Patients admitted to Kadimi Hospital with fracture distal end femur (distal $15 \mathrm{~cm}$ of femur)

a) All types of fracture lower end of femur (AO classification

A, B \& C). b) Only closed fractures were considered.

c) Paediatric patients and pathological fractures excluded.

\section{Inclusion Criteria}

1. Those patients who are of or above the age of $20 \mathrm{yrs}$ and managed surgically were included in the study

2. Patients presenting with distal femoral fractures with or without osteoporotic changes were included in the study

\section{Exclusion Criteria}

1. Patients with open distal femoral fractures

2. Children with distal femoral fractures in whom, growth plate is still open.

3. Patients lost in follow - up

4. Patients managed conservatively for other medical reasons.

5. Distal femoral fractures with neurovascular compromise

\section{Implant Used}

The plate and screws are manufactured from $316 \mathrm{~L}$ stainless alloy with gun drilling technique. The locking compression plates from 8 holed to 14 holed with $4.5 \mathrm{~mm}$ thickness that are anatomically precontoured are used.

\section{Preoperative Investigation}

Hemogram
Blood sugar level Blood urea level
Serum creatinine level
Liver function test
Blood group and Rh typing Bleeding time, clotting time

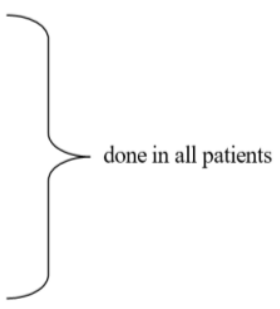

Chest X-ray PA view, Doppler if required, ECG, 2D echo and other investigations done as per requirement during the anaesthetic evaluation

Preoperative planning and preparation: Fractures were classified according to the AO-ASIF classification. Preoperative calculation was done on radiographs to ascertain the size of the plate, accurate size of locking, cortical and cancellous screws after subtraction of the magnification factor.

The limb to be operated was prepared. 1 gm of 3rd generation cephalosporin was injected the night before surgery and early morning on the day of surgery before shifting the patient to the operation theatre.

\section{Surgical Procedure}

Patient is placed supine on a table with a pillow below the knee, the entire injured extremity and ipsilateral iliac crest are prepared and draped. Tourniquet applied. Lateral incision is made parallel to the shaft of the femur, beginning at the Gerdy tubercle and extending proximally far enough to permit application. Longitudinal incision is made through the fascia lata, and extended distally into the iliotibial band.

More proximally, the fascia overlying the vastus lateralis muscle is incised and the muscle reflected anteriorly off the intermuscular septum. Minimal Stripping of soft tissue necessary for application of the plate and reduction of the articular surface is done. To preserve their vascularity, attempt is not made to expose and anatomically reduce comminuted anterior and metaphyseal fragments. Quadriceps mechanism and the patella are reflected medially to expose the entire lower end of the femur.

\section{Reduction of Condyles}

To aid in reduction of the condyle, a Steinmann was drilled into the lateral surface of the lateral condyle, if needed, a similar pin was placed in the medial condylar fragment. Using these pins as levers, manipulation and reduction of the two major condylar fragments was done to restore the articular surface and patellofemoral groove.

Two condyles were fixed together with multiple 2-mm Kwires. Medial and lateral condyles together were fixed with 6.5-mm cancellous screws directed lateral to medial, taking care not to interfere with the subsequent path of other cancellous screws of locking compression plate. Second screw was placed without a washer, slightly anterior and proximal to the first. K-wires used for temporary fixation was removed. 


\section{Reduction of the condyles to the femoral shaft}

When using the plate as a reduction aid, the compression screw draws the bone towards the plate and uses the contour of the plate to reduce the fracture in the coronal plane. Reduction of the fracture was assisted keeping folded pillow below the knee which prevented posterior angulation of distal fragment with manual traction. Once the fracture is reduced, supplemental locking screws were then added to create a fixed-angle construct. The compression mode was used to address reduced articular fractures through the plate, and in simple fracture patterns

\section{Criteria for union}

A fracture was considered united and fit for unprotected complete weight bearing in the absence of pain and presence of bridging callus in three cortices seen on anteroposterior and
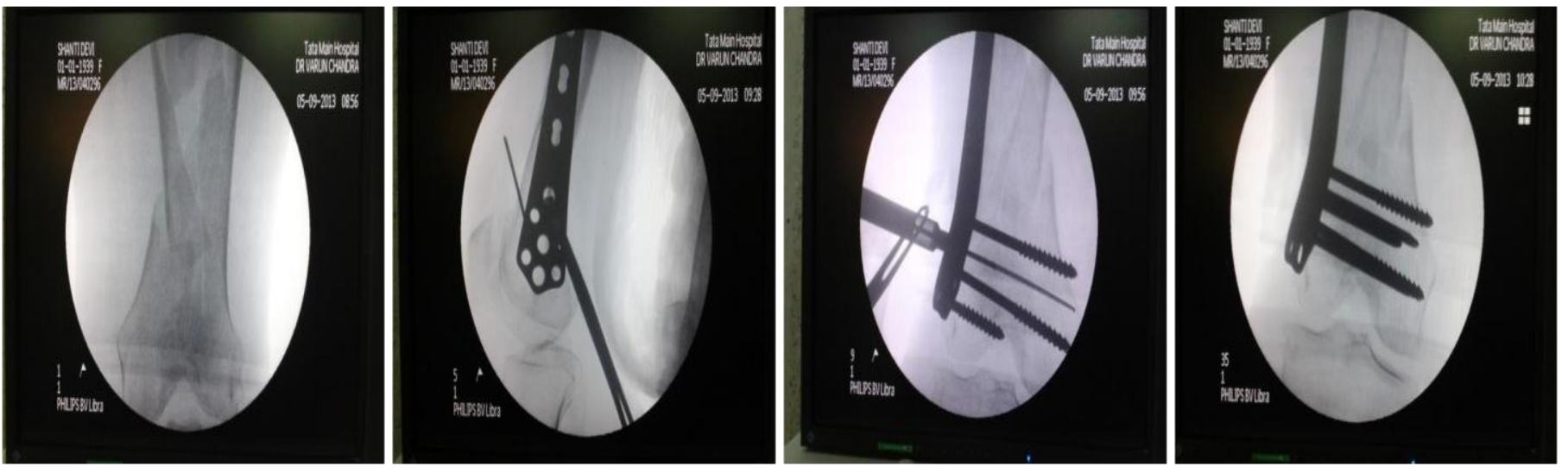

lateral radiographs. A fracture was considered radiologically un-united when there was absence of bone bridging or no evidence of progressive healing over a period of 3 months.

\section{Criteria for primary bone grafting}

Primary bone grafting used in cases of severe comminution as we were opening the fracture site and evacuating the fracture hematoma.

Adding primary autogenous bone grafting to the locked bridging constructs not only improves the biological environment with rapid callus formation, but also mechanically protects the construct - once integrated - against repetitive stresses that can cause varus collapse with loss of reduction, non-union and implant failure

\section{Surgical Steps}

\section{Case 1}
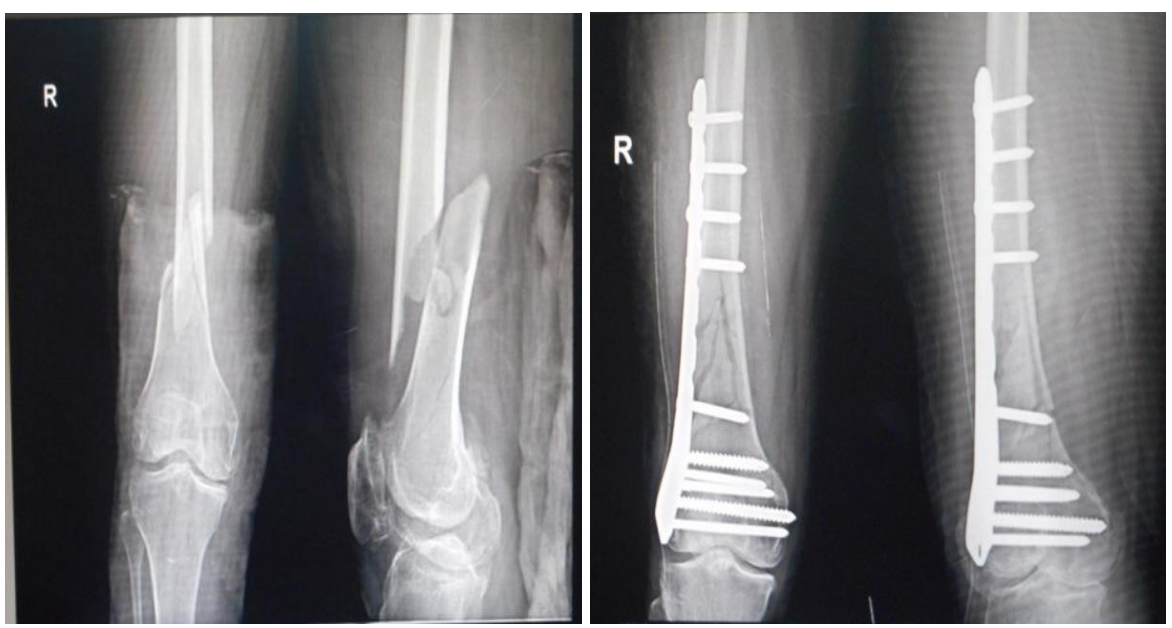

Case 2

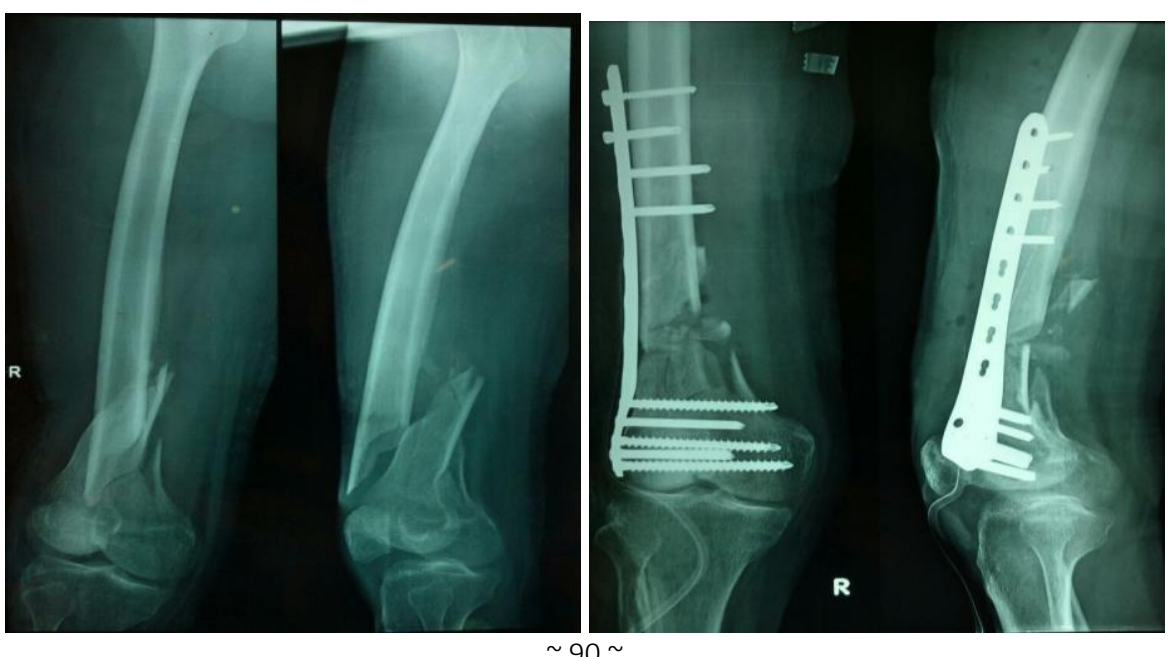


Case 3

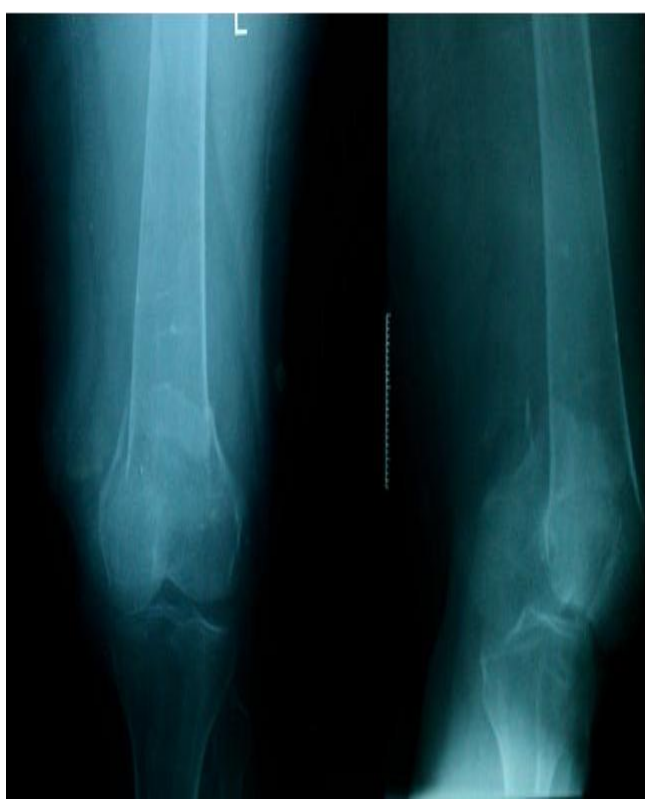

Post OP

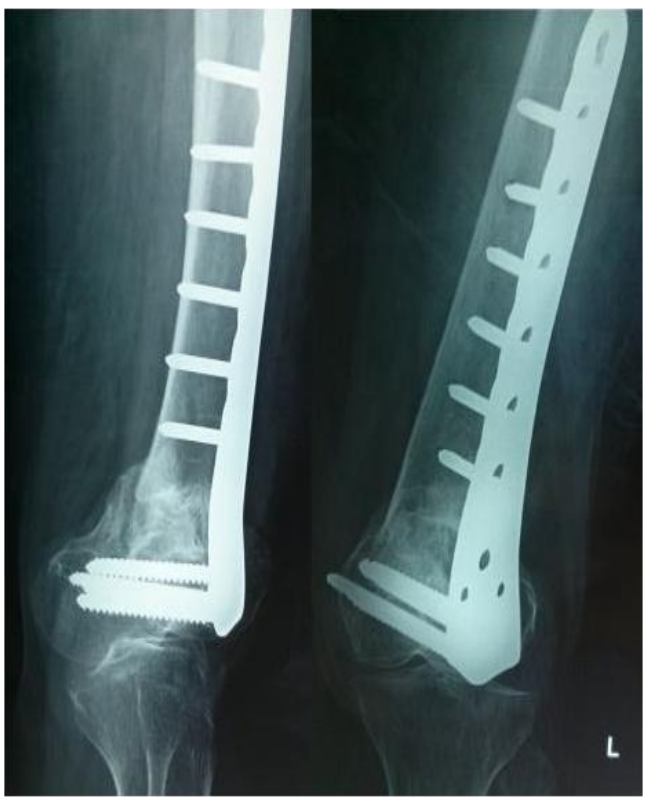

After 4 Months

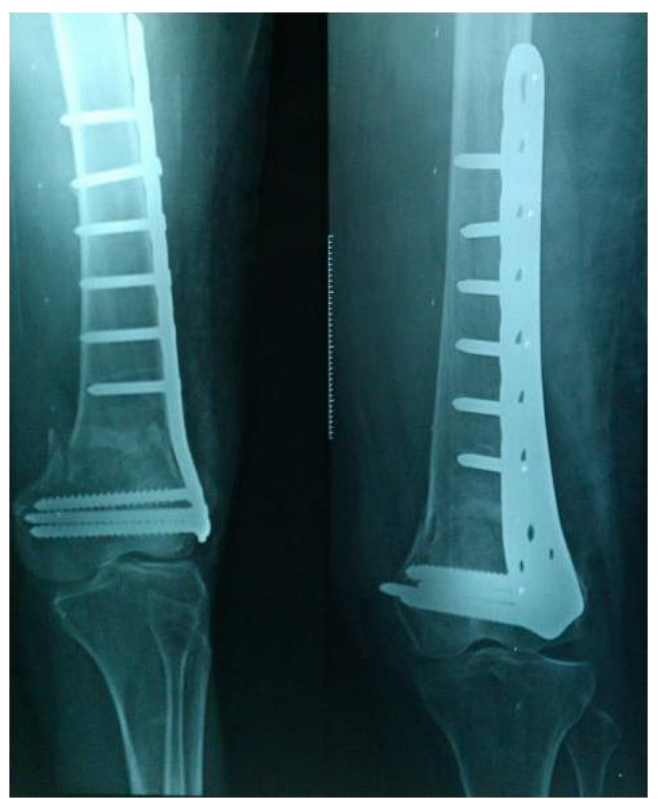

Post OP

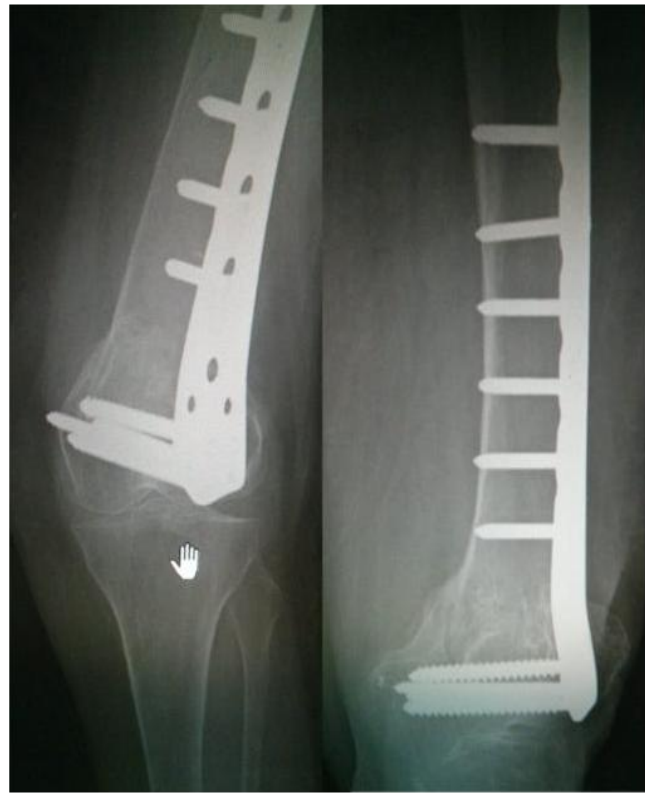

After 8 Months

\section{Clinical Pictures}

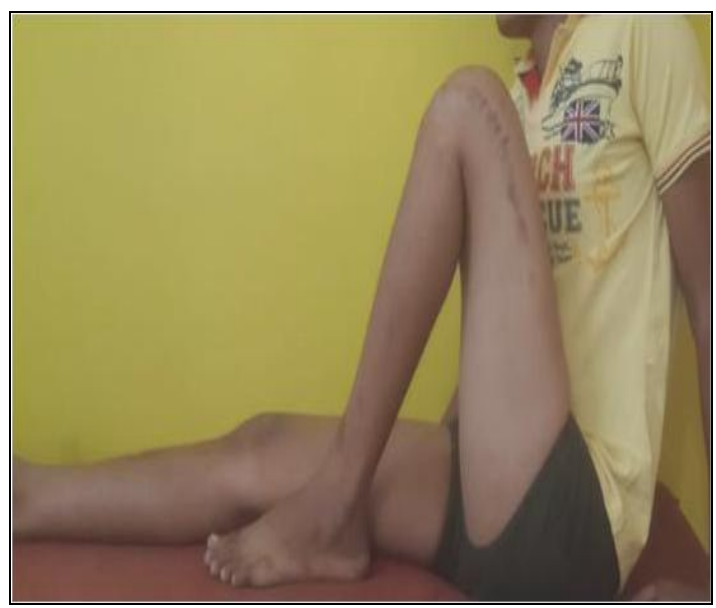

Flexion

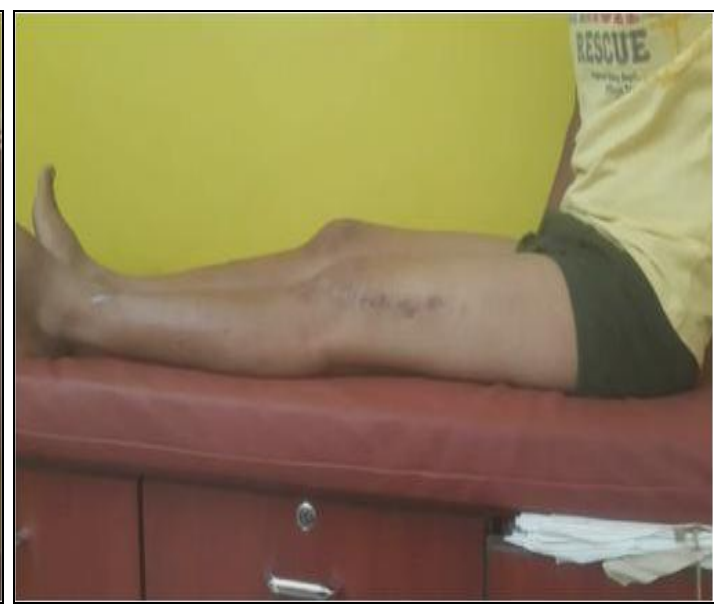

Extension 


\section{Postoperative}

Patients' vitals were monitored. Foot end elevation was given overnight, drain was monitored. Antibiotics were given as per the hospital protocol. Analgesics were given as per the patient compliance. Blood transfusion was given depending upon the preoperative general condition and intraoperative blood loss.

Mobilisation: Splints were removed and mobilization of the limb started on the 3rd or 4th day postoperatively. Mobilization with Non weight bearing was started from the first post-operative week till 6-8 weeks depending on the fracture pattern and then partial weight bearing after confirmation of beginning of healing process till fracture union.

Follow UP: All patients were followed up at 6th 10th 14th 18th week and 6 weeks thereafter till fracture union is noted. Subsequently at 6th month 9th month and 1 year. During follow up patients were assessed clinically, radiologically and functionally by NEERS criteria.

\section{Results}

In this study, 40 distal femoral fractures were treated using the distal femur locking compression plate. All cases were fresh, and all fractures were closed. Cases were followed up from a minimum of 6 months to maximum of 18 months.

Epidemiology: 26 patients were males and 14 patients were females. The median age was 43 years ranging from $20-75$ years. 22 patients were with fracture on right side 18 on left side.

Mechanism of injury: 30 of the fractures which include about 75 were caused by road traffic accidents and 4 were due to accidental $\mathrm{f}$ alls making $10 \%$ of cases, 2 were due to assault and 4 cases were due to other causes. All fractures were fresh and closed

\section{Classification}

Type of Fracture (MULLER'S)

\begin{tabular}{|c|c|c|}
\hline Muller's type & No. Of cases & Percentage \\
\hline A1 & 8 & $20 \%$ \\
\hline A2 & 4 & $10 \%$ \\
\hline A3 & 4 & $10 \%$ \\
\hline B1 & 0 & $0 \%$ \\
\hline B2 & 0 & $0 \%$ \\
\hline B3 & 0 & $0 \%$ \\
\hline C1 & 3 & $6.60 \%$ \\
\hline C2 & 12 & $30 \%$ \\
\hline C3 & 9 & $23.30 \%$ \\
\hline TOTAL & 40 & $100 \%$ \\
\hline
\end{tabular}

\section{Perioperative}

All patients were treated with open reduction and internal fixation using Locking Compression Plate.

All patients were operated within 10 days. Depending on the medical and surgical co- morbidities the injury-surgery interval lasted from 2 days to 10 days. Average time duration of surgery was 105 minutes with shortest duration being 90 min and longest being $130 \mathrm{~min}$. The size of plate was selected based on the type of fracture. 7 to 9 holed plates were used more commonly for lower end of femur.

Bone grafting: 8 of the cases had bone grafting of which 6 had primary bone grafting and two patients had secondary bone grafting done at the end of 5 months for delayed union and both cases went on to union after secondary bone grafting.

Union: Of 40 patients, 11 Patients (26.66\%) showed radiological union within 15 weeks and $85 \%$ cases united within 22 weeks. 1patient had implant failure. average time taken for union was 15.25 weeks. 2 cases went for delayed union and 3 cases went for non-union.

Range of motion: Average flexion in this study was $109^{0}$ with $50 \%$ patients having knee range of motion more than $100^{\circ}$. Average knee extensor lag in this study was 2.4 degrees with only 5 patients with lag more than $5^{0}$

\section{Complications}

\begin{tabular}{|c|c|c|}
\hline Complications & $\begin{array}{c}\text { No. Of } \\
\text { cases }\end{array}$ & Percentage \\
\hline Superficial infection & 2 & $5 \%$ \\
\hline Deep infection & 2 & $5 \%$ \\
\hline Delayed union & 2 & $5 \%$ \\
\hline Non-union & 4 & $10 \%$ \\
\hline Plate Backout & 0 & $0 \%$ \\
\hline $\begin{array}{c}\text { Implant failure - screw /plate } \\
\text { breakage }\end{array}$ & 1 & $2.5 \%$ \\
\hline Varus/ Valgus of >5 degrees & 2 & $5 \%$ \\
\hline Extension lag > 5 degrees & 5 & $12.5 \%$ \\
\hline
\end{tabular}

Non-union: 4 out of 40 cases went for non-union out of which 2 were due to deep infection that was managed with debridement and Ilizarov ring fixator and the other 2 were gap non union which are being planned for revision.

Limb length discrepancy: Of 40 patients, 9 had shortening 4 patients with up to $1 \mathrm{~cm}, 3$ cases with $1-2 \mathrm{~cm}$ and 2 cases over $2 \mathrm{~cm}$ shortening.

Malalignment: In this study, only 2 patients had malalignment of over 5 degrees and 4 patients had less than 5 degrees of mal-alignment.

Infection: 2 of the patients had superficial surgical site infection which was controlled before the patients were discharged from the hospital. 2 patients had deep infections and were treated with debridement and antibiotics but went on for non- unions and were managed with Ilizarov ring fixator.

Functional Outcome: Functional outcome was measured using NEER's scoring system and was done at the end of 8-12 months (average of 6 months).

\section{Functional Results (Based On Neer Scoring)}

\begin{tabular}{|c|c|c|}
\hline Neer grade & No. of cases & Percentage \\
\hline Excellent & 23 & $57.7 \%$ \\
\hline Good & 10 & $25 \%$ \\
\hline Fair & 3 & $7.5 \%$ \\
\hline Poor & 4 & $10 \%$ \\
\hline Total & 40 & $100 \%$ \\
\hline
\end{tabular}

Results are analyzed in charts and tables below using statistical methods of analysis. The data collected in the present study was analyzed statistically by SPSS version 17 software appropriate statistical tests were used to determine 
the efficacy of outcome such as chi-square test, p-values for significance along with means and standard deviations for descriptive studies wherever it was appropriate.

\section{Discussion}

All cases studied in the series were closed fractures. 26 patients were males and 14 patients were females. The median age was 40 years ranging from 20 - 75 years. 30 of the fractures were caused by road traffic accidents and 4 were due to fall and 2 were due to assault and 4 were due to other causes. 22 patients were with fracture on right side and 18 on left side.

Of the mechanisms of injury, all the younger patients (2nd to 4th decade) had a high energy trauma like the road traffic accident in our demography or fall from height or assault, and trivial trauma was the most common cause of fracture in older age group.

Primary bone grafting was used in $8 \operatorname{cases}(20 \%)$ to promote union in cases of comminuted fractures (A2, A3, C2, and C3) as all the cases were reduced by open reduction and internal fixation as LISS technique was not used, though we used minimally invasive approach in certain cases for proximal screw placement.

In this study, primary bone grafting was used in cases of severe comminution in selected patients as we were opening the fracture site and evacuating the fracture hematoma and also secondary bone grafting was done in 2 cases of delayed union.

We conclude that bone grafting is required primarily usually in cases of severe comminution and where metaphyseal bone defect is present, and secondarily in cases where delayed union and implant failure were present. Moreover use of locking plate reduces the need for bone grafting also.

Non union occurred due to gross comminution at the fracture site, infection inhibiting callus formation and interference with the fracture hematoma as our procedure is open reduction and internal fixation rather than minimally invasive surgery. Non-union was noted in $10 \%$ of patients which treated with bone grafting. Hoffmann et al. ${ }^{[6]}$ had $18 \%$ nonunion rate, zlowodzki et al. ${ }^{[7]}$ had $5.5 \%$ non-union rate.

In the present study $26.66 \%$ off cases united by 15 weeks and overall $77.5 \%$ cases united by 18 weeks of time. Mean radiological fracture union time was 16.2 weeks. In $\mathrm{R}$. pascarella et al. ${ }^{[8]}$ study mean radiological fracture union time was 16.3 weeks

Delayed union occurred in 2 cases due to premature weight bearing against medical advice and comminution at the fracture site. $5 \%$ of cases reported delayed union. In Rajnish $\mathrm{R}$ menon et al. ${ }^{[9]}$ study $2 \%$ delayed union was reported

Complication rate is $15 \%$ (10\% non-union and 5\% delayed union) and 1 case $(2.5 \%)$ of implant failure occurred at the end of 8 months. Schutz et al. ${ }^{[10]}$ reported $4.5 \%$ implant failure rate. In our study it was a case of comminuted supracondylar fracture Muller type C3, which went for a complication of screw breakage and implant failure. On analyzing it retrospectively we believe the cause for implant failure was shorter plate length (6 holed) and severe metaphyseal comminution which lead to gap non-union. The case is being planned for exchange plating with a longer plate with bone grafting

There were 4 cases of non-union, two of which were found to be due to deep infection. They were managed with implant removal, wound debridement with application of local antibiotic beads and application of Ilizarov ring fixator

We would like to conclude that complete reduction of communited distal femoral fractures are difficult. It is advisable to assess communited distal femoral fractures before considering for surgery and predict varus malalignment. In case malalignment is predicted, other modalities like medial additional plating, using maximum number of screws in distal fragment, bone grafting and delayed full weight bearing are advisable. The chances of malalignment/malunion in distal femur locked plating in inexperienced hands are higher as the reduction of communited fractures is difficult.

Other factors contributing to malunion are unstable fixation, inadequate fixation and early weight bearing on part of the patients. Malalingment occurred as a result of improper reduction intraoperatively. Other factors contributing to malalignment were comminution and use of inadequate locking plate length due to which the fracture went into varus. In 1 distal femoral fracture, varus malalignment occurred as a result of early weight bearing done against advice.

At a mean follow-up of 10 months, the mean knee flexion was 3-109 degrees. Yeap et al. ${ }^{[1]}$ study shows mean knee range of motion 1-107degrees.

Physiotherapy was started immediately the next day after surgery with active toe movements, static quadriceps exercises. Quadriceps strengthening exercises were started after suture removal with strict non weight bearing gait with walker. ROM were checked at every visit and vigorous physiotherapy was advised for the patients.

In this study of 40 patients 23 patients (57.5) show excellent results, 10 patients (25.7) show good results, 3 patients (3) show fair results, 4 patients (10) show poor results.

\section{Limitations}

Limitations of our study are that the minimum follow up of 12-18 months is only enough to comment on healing and midterm functional results. Development of degenerative joint disease which would be interesting with such a high rate of intra-articular fractures (consisting of $60 \%$ of cases) cannot be commented upon in this study.

In this study, open injuries where LCP was used after primary debridement were not included. So, this cohort does not assess the functional outcome of LCP in cases with damage to soft tissues following open wounds.

Another important determinant in the study is that most of the patients in this cohort are illiterates and daily labourers who cannot afford regular and proper physiotherapy following surgery which we believe, has a definite role in functional outcome.

Finally, the study cohort is relatively small with only 40 cases meeting the inclusion criteria and hence further studies with multicentric large scale prospective studies are needed for further evaluation of outcome.

\section{Conclusion}

LCP is a good implant to use for fractures of the distal femur. However, accurate positioning and fixation are required to produce satisfactory results. LCP is an excellent implant in Type $\mathrm{A}$ and $\mathrm{C}$ and also in osteoporotic fractures. Early results are encouraging but long term studies are needed to prove a comparable or better outcome so that the technique can become a part of the armamentarium of the orthopaedic trauma surgeon. MIPO technique preserves most of the osseous vascularity and fracture hematoma thus providing for a more biological repair. There is rapid fracture consolidation due to preserved vascularity. There are fewer incidences of delayed union and non-union. There is a decreased need for bone grafting and incidence of infection is less due to limited exposure. 


\section{Recommendations}

1. We recommend use of LCP in all distal femur fractures as it is far superior to other implants like DCS.

2. Locking plates when used should be used with minimally invasive methods like MIPPO or LISS which gives better functional results as soft tissue dissection is kept to minimum.

3. We recommend use of $\mathrm{LCP}$ in type $\mathrm{A}$ and type $\mathrm{C}$ fractures and in osteoporotic distal femur fractures.

4. Intraoperative attention should be given to restore alignment in all planes.

5. We recommend aggressive care and management in patients above 50 years of age as there are chances of having associate medical problems

6. We recommend judicious use of bone grafting in comminuted distal femur fractures to improve outcome and union rates.

\section{References}

1. Arneson TJ, Melton LJ 3rd, Lewallen DG, et al. Epidemiology of diaphyseal and distal femoral fractures in Rochester, Minnesota, 1965-1984. Clin Orthop. 1988; 234:188-94.

2. Schandelmaier P, Partenheimer A, Koenemann B, Grun OA, Krettek C. Distal Femoral Fractures and LISS Stabilization, Injury. 2001; 32(SC):55-63

3. Michael Z, Mohit B, et al. Operative treatment of acute distal femur fractures: systematic review of 2 comparative studies and 45 case series (1989-2005). J Orthop Trauma. 2006; 20:366-71.

4. Heather V, Theresa H, John S, et al. Failure of LCP condylar plate fixation in the distal part of the femur. $\mathrm{J}$ Bone Joint Surg. 2006; 88-A:846-53.

5. Kregor PJ, Stannard JA, Zlowodzki M, et al. Treatment of distal femur fractures using the less invasive stabilization system: surgical experience and early clinical results in 103 fractures. J Orthop Trauma. 2004; 18:509-520.

6. Hoffmann MF, Jones CB, Sietsema DL, Tornetta P 3rd, Koenig SJ. Clinical outcomes of locked plating of distal femoral fractures in a retrospective cohort. J Orthop Surg Res. 2013; (27)8-43.

7. Zlowodzki M, Bhandari M, Marek DJ, Cole PA, Kregor PJ. Operative treatment of acute distal femur fractures: systematic review of 2 comparative studies and 45 case series (1989 to 2005). J Orthop Trauma. 2006; 20(5):366371.

8. Pascarella R, Bettuzzi C, Bosco G, et al. Results in treatment of distal femur fractures using polyaxial locking plate. Strategies in Trauma and Limb Reconstruction. 2014; 9(1):13-18.

9. Menon RR, Subramanian V. Functional outcome of distal femoral fractures treated by minimally invasive surgery using locking condylar plate. Kerala J Orthop. 2014, 27(1).

10. Schutz M, Muller M, Regazzoni P, et al. Use of the Less Invasive Stabilization System (LISS) in patients with distal femoral (AO33) fractures: a prospective multicenter study. Arch Orthop Trauma Surg. 2005; 125(2):102-8.

11. Yeap EJ, Deepak AS. Distal Femoral Locking Compression Plate Fixation in Distal Femoral Fractures: Early Results. Malaysian Orthopaedic Journal. 2007; 1(1):12-17. ISSN 19852533 